\title{
VIBRATIONAL COMMUNICATION OF NINE CALIFORNIA STONEFLY (PLECOPTERA) SPECIES
}

\author{
John B. Sandberg1
}

\begin{abstract}
The drumming signals of 9 California stonefly species from 8 families are reported. Signal interval patterns of the individual interbeat, intragroup, and intergroup intervals are graphed and used to determine signal type (monophasic, varied beat-interval, diphasic, and grouped). Signals of Kathroperla takhoma Stark \& Surdick are described for the first time. New signal characters and signals from additional locations are described for 8 species: Bolshecapnia maculata (Jewett), Calineuria californica (Banks), Doroneuria baumanni Stark \& Gaufin, Megaleuctra complicata Claassen, Nemoura spiniloba Jewett, Oemopteryx vanduzeea (Claassen), Pteronarcys princeps Banks, and Sierraperla cora Needham \& Smith.
\end{abstract}

Resumen.-Se reportan las señales de tamborileo de 9 especies pertenecientes a 8 familias de plecópteros de California. Los patrones de los intervalos entre pulsaciones individuales, intragrupos e intergrupos son graficados y utilizados para determinar la clase de señal (monofásica, de intervalos variados de pulsación, difásica y agrupadas). Se describen por primera vez las señales de Kathroperla takhoma Stark y Surdick. Presento la descripción de nuevos caracteres de señales, así como nuevas señales, de poblaciones en nuevos sitios para 8 especies: Bolshecapnia maculata (Jewett), Calineuria californica (Banks), Doroneuria baumanni Stark y Gaufin, Megaleuctra complicata Claassen, Nemoura spiniloba Jewett, Oemopteryx vanduzeea (Claassen), Pteronarcys princeps Banks y Sierraperla cora Needham y Smith.

Intersexual vibrational communication (or drumming) in Plecoptera is a species-specific mate-location behavior initiated by actively searching males. Drumming behavior takes place mostly on branches, leaves, or bark of riparian vegetation. The primarily percussive signals are some of the most complex signals known in insects (Stewart 2001). Drumming signals are transmitted by abdominal-substrate tapping or rubbing and are presumably received through the substrate by antennae and tarsi. Searching males produce call signals $\left(\hat{\delta}_{\mathrm{C}}\right)$, and only virgin and stationary females answer ( + ) (Stewart 1994, 2001, Stewart and Sandberg 2006). Drumming and the associated aggregation and movement behaviors of both sexes (Stewart 1994) facilitate mate-location in complex riparian habitats.

Percussive drumming ranges in complexity from simple monophasic signals, with approximately even interbeat intervals, to more complex varied beat-interval, grouped, and diphasic signals. Male rub calls are produced by quickly dragging the ventral and posterior abdomen along the substrate surface and can include additional percussive beats (Maketon and Stewart 1988). Tremulation calls are produced by quick, rocking body movements without abdominalsubstrate contact, and, when recorded, sound similar to percussive monophasic signals (Alexander and Stewart 1997). Some of the most complex calls are combinations of signal types such as a diphasic signal followed by a series of grouped signals (Stewart et al. 1991). Intersexual communication ranges from simple (or sequenced) 2-way duets $\left(\hat{o}_{\mathrm{C}^{-}}^{-}+\right)^{2}$ to complex 3way exchanges with the male producing a second signal type called the response $\left(\hat{o}_{\mathrm{C}}-q-\hat{O}_{\mathrm{R}}\right)$. Grouped 4-way or longer call-and-answer sequences and symphonic signals with interspersed female answers lasting approximately 10-20 seconds are some of the most complex exchange types in Plecoptera (Maketon et al. 1988, Sandberg and Stewart 2005).

This study was undertaken to add to the knowledge of drumming behavior in Nearctic stoneflies by recording and analyzing signals of reared-virgin and field-collected adults from 8 families of California stoneflies (Capniidae, Chloroperlidae, Leuctridae, Nemouridae, Peltoperlidae, Perlidae, Pteronarcyidae, and Taeniopterygidae).

\section{Methods}

Virgin adults were reared from mature nymphs obtained with a kicknet, and wild

${ }^{1}$ Aquatic Bioassessment Lab - California Department of Fish and Game \& CSUC Research Foundation, California State University, Chico, CA 95929-0555. E-mail: jsandberg@csuchico.edu 
adults were collected using a beating sheet and aspirator from the following perennial and nonperennial streams (abbreviations in parentheses are used in Table 2 after the species listing and in interval pattern figures).

(1) Bolshecapnia maculata, California, Butte Co., Oregon Gulch (OG), Oregon City, Oregon Gulch Rd., $39.59373^{\circ} \mathrm{N},-121.52986^{\circ} \mathrm{W}$, 23-II- \& 01-III-2008 ( ơ ơ field-collected, $\uparrow$ reared). (2) Calineuria californica, California, Butte Co., Big Chico Creek (BCC), Big Chico Creek Ecological Reserve, $39.86909^{\circ} \mathrm{N}$, $-121.70760^{\circ} \mathrm{W}$, near $\mathrm{N}$ boundary, $24-\mathrm{V}-2009$ ( 0 ô field-collected, क reared). (3) Doroneuria baumanni, California, Tehama Co., Big Chico Creek (BCC), Hwy. 32 bridge, $2.3 \mathrm{mi}$. $(3.7 \mathrm{~km}) \mathrm{N}$ Lomo (Humboldt Rd. intersection), $40.06321^{\circ} \mathrm{N},-121.60497^{\circ} \mathrm{W}, 24-\mathrm{IX}-$ 2007 ( 9 and ôे fold-collected). (4) Kathroperla takhoma, California, Trinity Co., Clear Creek and adjacent tributaries (CCT), Hwy. 36, $2.6 \mathrm{mi}$. (4.2 km) East Forest Glen Campground, $40.3743^{\circ} \mathrm{N},-123.36347^{\circ} \mathrm{W}, 09-\mathrm{V}$ 2009 ( $\widehat{0}$ ô field-collected). (5) Megaleuctra complicata Claassen, California, Humboldt Co., EF Willow Creek (EWC), Hwy. 299, East Fork Campground, spring seeps, 0.5 mi $(0.8 \mathrm{~km})$ from Hwy. $299,40.90798^{\circ} \mathrm{N}$, $-123.70723^{\circ} \mathrm{W}, 20-\mathrm{IV}-2009$ ( ฮิ ฮิ reared). (6) Nemoura spiniloba Jewett, California, Butte Co., Campbell Creek and tributary (CC\&T), North Table Mt. Wildlife Area, Cherokee Rd., 5 mi. $(8 \mathrm{~km}) \mathrm{S}$ Cherokee, $39.58897^{\circ} \mathrm{N}$, $-121.55896^{\circ} \mathrm{W}, 17-$-II- \& 03-III-2007 (oิ o reared), 23-II-2008 ( $\delta \hat{\delta}$ reared and collected, $q$ reared). (7) Oemopteryx vanduzeea (Claassen), California, Butte Co., Campbell Creek and tributary (CC\&T), North Table Mt. Wildlife Area, Cherokee Rd., 5 mi. (8 km) S Cherokee, $39.58897^{\circ} \mathrm{N},-121.55896^{\circ} \mathrm{W}$, 02-II- \& 17-II-2007 ( $q$ and $\hat{o} \delta$ ô reared), 23-II\& 08-III-2008 ( के ठิ reared); Oregon Gulch (OG), Oregon Gulch Rd., Oregon City, $39.59373^{\circ} \mathrm{N},-121.52986^{\circ} \mathrm{W}, 23-\mathrm{II}-\&$ 01-

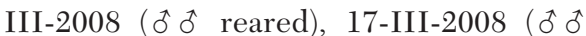
reared). (8) Pteronarcys princeps Banks, California, Butte Co., Little Butte Creek (LBC), Skyway Ave., $0.1 \mathrm{mi} .(0.16 \mathrm{~km}) \mathrm{S}$ Toadtown, $39.88554^{\circ} \mathrm{N},-121.59137^{\circ} \mathrm{W}, 28-\mathrm{I}-2007$ (우 우 and $\hat{o}$ ô reared). (9) Sierraperla cora (Needham \& Smith), California, Butte Co., Butte Creek (BCCH), Cherry Hill Campground (Humboldt Rd.), 9 mi. (14.5 km) NE Lomo (Hwy. 32), $40.10226^{\circ} \mathrm{N},-121.49911^{\circ} \mathrm{W}, 01-$ IV- \& 20-IV-2007 (ô ô reared, of $q$ reared and collected).
Six hundred ninety-nine drumming signals were recorded in the laboratory using methods of Sandberg and Stewart $(2003,2006)$, at a range of temperatures between $19.4-22.8{ }^{\circ} \mathrm{C}$ and with the use of incandescent light. In several recordings of Nemoura spiniloba and Sierraperla cora, several females answered the playback of previously recorded male calls through speakers approximately $3.5 \mathrm{~m}$ from the recording chambers. Only the answers were analyzed for these recordings.

Drumming descriptions include descriptive text, general characters, detailed characters (Tables 1-3), and oscillogram screen-captures of typical signals (Figs. 1-5, 12-17). A new character utilized in this study, the interval pattern, is included to describe the variation observed within (or throughout) an entire signal and among the successive signals of an individual or group. The interval pattern or the relative change in beat or group intervals over time is described using boxand-whisker charts showing the mean, standard deviation, and range of individual signal intervals (Figs. 6-11, 18-25). These charts display time intervals between the beats of monophasic, varied beat-interval, and diphasic signals (interbeat intervals); time intervals between grouped beats (intragroup intervals); and time intervals between groups (intergroup intervals), all measured in milliseconds. Interval pattern written descriptions were based on the mean (dashed line in the figures), and variation among individual intervals was documented with standard deviation (box) and range (vertical lines).

\section{RESUlTS}

All numbers of signal beats and time intervals presented in the following descriptions are expressed as $\bar{x} \pm \mathrm{SD}$, with range in parentheses. Abbreviations: $\mathrm{i}=$ interval and $\mathrm{d}=$ days old. For wild (field-collected) stoneflies, age refers to successive days held in captivity until recordings were made.

\section{CAPNIIDAE}

BOLSHECAPNIA MACULATA. - The 4-way exchanges of this species were not typical because males and females consistently called and answered twice. Typical 4-way exchanges are aborted 5-way male-female signals where the male did not produce a response signal. In 3way, 5-way, or greater odd-numbered exchanges of other species, the last male signal is usually 
TABLE 1. General signal characters of 8 previously published descriptions and congener Kathroperla perdita. Previous descriptions are within parentheses when updated by the current study (followed by a dagger symbol). Abbreviations: Abdomen $(\mathrm{Abd})$ with hammer $(\mathrm{H})$, lobe $(\mathrm{L})$, knob $(\mathrm{K})$, or with no specialized structure $(\mathrm{N})$; male call $\left(\hat{\delta}_{\mathrm{C}}\right)$, female answer $(q)$, and male response $\left(\hat{\delta}_{R}\right)$; monophasic $(M)$, varied beat-interval $(\mathrm{VB})$, diphasic $(\mathrm{D})$, grouped $(\mathrm{G})$, combination $(\mathrm{C})$ and rub (R). These descriptions and their detailed characters listed in Table 3 are provided for comparison to the current study.

\begin{tabular}{|c|c|c|c|c|c|c|}
\hline Species & Abd & $\hat{o}_{\mathrm{C}}$ & q & $\hat{o}_{\mathrm{R}}$ & Exchange & Citation - State \\
\hline Bolshecapnia maculata & $\mathrm{L}$ & (M) $\mathrm{M} \& \mathrm{VB}^{\dagger}$ & $\mathrm{VB}^{\dagger}$ & & 3- to 4 -way ${ }^{\dagger}$ & Stewart et al. 1991 - CA \\
\hline Calineuria californica & $\mathrm{H}$ & $\mathrm{R}$ & $(\mathrm{M}) \mathrm{VB}^{\dagger}$ & M & 3-way & Stewart et al. 1982 - CA \\
\hline Doroneuria baumanni & $\mathrm{H}$ & $\mathrm{R}$ birub & $(\mathrm{M}) \mathrm{VB}^{\dagger}$ & & 2-way & Maketon \& Stewart - CA \\
\hline Kathroperla perdita & $\mathrm{N}$ & (D) $\mathrm{G}^{\dagger}$ & & & & Stewart \& Zeigler 1984 - OR \\
\hline Megaleuctra complicata & $\mathrm{N}$ & $\mathrm{G}$ & & & & Stewart \& Sandberg 2004 - OR \\
\hline Nemoura spiniloba & $\mathrm{N}$ & (D) $\mathrm{C} \mathrm{VB}-\mathrm{G}^{\dagger}$ & G & G & 2- to 4-way & Stewart et al. 1991 - CA \\
\hline Oemopteryx vanduzeea & $\mathrm{L}$ & M & $\mathrm{M}^{\dagger}$ & $\mathrm{M}^{\dagger}$ & & Stewart et al. 1991 - CA \\
\hline Pteronarcys princeps & $\mathrm{L}$ & $(\mathrm{M}) \mathrm{VB}^{\dagger}$ & $(\mathrm{M}) \mathrm{VB}^{\dagger}$ & $(\mathrm{M}) \mathrm{VB}^{\dagger}$ & 3-way & Zeigler \& Stewart 1985 - CA \\
\hline Sierraperla cora & $\mathrm{K}$ & $(\mathrm{M}) \mathrm{VB}^{\dagger}$ & M & M & 3- to 5-way & Zeigler \& Stewart 1985 - CA \\
\hline
\end{tabular}

†Characters updated in this report

the response signal, which differs from the call in interbeat interval or number of beats. The male response normally indicates the end of an intersexual drumming exchange, except the highly complex signals of Nemoura spiniloba (Stewart et al. 1991), where females produce 2 similar monophasic answers, one before and one after the response. The signals of B. maculata are similar to $N$. spiniloba because they lack an exchange-ending male response signal but differ in that the second female answer has fewer mean beats per signal and longer intervals.

Seventy-two first calls $\left(\hat{\partial}_{1}\right), 72$ first answers $\left(q_{1}\right), 72$ second calls $\left(\delta_{2}\right)$, and 56 second answers $\left(q_{2}\right)$ were recorded from two 1-d males and two 1-d females at $19.4-20.6{ }^{\circ} \mathrm{C}$. Males signaled with monophasic first calls and varied beat-interval second calls. Females signaled with varied beat-interval first and second answers with 3-way $(n=16)$ or 4-way $(n=56)$ exchanges (Fig. 1). The second male call differed from the first by having slightly more mean beats and longer interbeat intervals (Table 2). Female second answers differed from the first by having fewer beats and slightly longer intervals. The first call signals had $5.2 \pm 0.5$ beats (4-6) with intervals of $32.6 \pm 1.6 \mathrm{~ms}$. The second call signal had $6.0 \pm 1.1$ beats (3-8) with intervals of $49.8 \pm 8.4 \mathrm{~ms}$. Call durations were ${ }_{1} 138.2 \pm$ $14.8 \mathrm{~ms}(95.8-168.0)$ and $\mathrm{o}_{2} 249.1 \pm 58.6 \mathrm{~ms}$ (102.6-366.4). The number of beats per first female answer signal was $5.5 \pm 1.6(3-9)$ with mean intervals of $54.2 \pm 14.3 \mathrm{~ms}$ (Fig. 1, Table 2 ), and the second answer had $2.2 \pm 0.8$ beats (1-5) with $72.9 \pm 15.8 \mathrm{~ms}$ intervals. The majority of multibeat second answer signals (40 out of 46) had varied beat-interval patterns. Answer durations were ${ }^{+}{ }_{1} 241.6 \pm 78.6 \mathrm{~ms}$ (106.2-
$438.5)$ and $q_{2} 106.2 \pm 44.6 \mathrm{~ms}$ (56.6-297.2). Exchange intervals between 3 -way and 4-way calls and answers were: $\hat{\delta}_{1}{ }^{-}{ }_{1}{ }_{1} 292.0 \pm 26.2 \mathrm{~ms}$ (Table 2); $\stackrel{+}{1}_{1}-\hat{O}_{2} 308.8 \pm 37.3 \mathrm{~ms}(167.9-374.9)$; and $\hat{o}_{2}{ }^{-} q_{2} 300.4 \pm 111.5 \mathrm{~ms}(87.1-496.1)$. The 3-way signal durations were $1194.2 \pm 90.0 \mathrm{~ms}$ (1038.9-1315.0, $n=16$ ), and 4-way signal durations were $1627.5 \pm 157.4 \mathrm{~ms}(1370.0-1873.7$, $n=56)$.

The first and second call and answer mean interval patterns increased; however, the first call increase was minimal (Fig. 6). The first call $\left(\hat{o}_{1}\right)$ interval pattern increased slightly from 31.4 $\mathrm{ms}$ (i1) to $35.6 \mathrm{~ms}$ (i5), with a 4.2-ms interval difference, and the second call $\left(\hat{\sigma}_{2}\right)$ pattern increased from $42.7 \mathrm{~ms}$ (il) to $74.0 \mathrm{~ms}$ (i7), with a 31.3-ms interval difference. The first female answer $\left(q_{1}\right)$ interval pattern increased from 43.7 $\mathrm{ms}$ (i1) to $86.4 \mathrm{~ms}$ (i8) with an interval difference of $42.7 \mathrm{~ms}$, and the second answer $\left(q_{2}\right)$ pattern increased slightly from $69.9 \mathrm{~ms}$ (i1) to 78.9 ms (i2) with a 9.0-ms interval difference. The last 2 intervals decreased to $67.7 \mathrm{~ms}$ (i3) $(n=$ 1) and then increased to $102.7 \mathrm{~ms}$ (i4) $(n=1)$.

The ${ }^{*}{ }_{1}$ raw interval data (i1-i5) from 72 signals were variable and did not all exhibit consistently increasing patterns, and the mean interval difference was $4.2 \mathrm{~ms}(35.6-31.4=4.2 \mathrm{~ms})$. Thus, the $\delta_{1}$ pattern was interpreted as a monophasic signal with approximately even intervals (Stewart and Sandberg 2006). However, varied beat-interval signals are defined as having variable rhythm or interbeat intervals (Stewart and Sandberg 2006), and the ( $\left.\hat{\partial}_{1}\right)$ call pattern could alternately be interpreted as this signal type with increasing mean intervals. Because these original definitions are vague and are based on outdated storage oscilloscope 
TABLE 2. Detailed signal characters of 9 California Plecoptera species recorded in 2007-2009. Male call ( $\widehat{\delta}_{\mathrm{C}}$ ), female component (VB). Total number of male rubs, intergroup interval (ms), rub duration (ms), and inter-rub interval (ms) are signal characters.

\begin{tabular}{|c|c|c|c|c|c|c|c|c|}
\hline \multirow[b]{2}{*}{$\begin{array}{l}\text { Species, water body } \\
\text { (Temperature, age) }\end{array}$} & \multicolumn{3}{|c|}{$\frac{\text { Individuals }}{\text { Signals }}$} & \multicolumn{2}{|c|}{$\begin{array}{lc}\text { Groups } & \text { Beats } \\
\text { per call } & \text { per group } \\
(\text { Range/[Avg } \pm S D])\end{array}$} & \multicolumn{3}{|c|}{$\begin{array}{l}\text { Total \# beats-rubs }{ }^{\mathrm{a}} \text { / signal } \\
\quad(\text { Range } /[\text { Avg } \pm \mathrm{SD}])\end{array}$} \\
\hline & $\hat{\sigma}_{\mathrm{C}} / \hat{\sigma}_{1}$ & $\begin{array}{l}q_{1} q_{1} \\
q_{2}\end{array}$ & $\sigma_{R}$ & $\delta_{\mathrm{C}}$ & $\sigma_{\mathrm{C}}$ & $\begin{array}{c}\hat{\sigma}_{\mathrm{C}} / \hat{\sigma}_{1} \\
\hat{\sigma}_{2}\end{array}$ & $\begin{array}{l}q_{/} q_{1} \\
q_{2}\end{array}$ & $\hat{\sigma}_{\mathrm{R}}$ \\
\hline \multirow{4}{*}{$\begin{array}{l}\text { B. maculata, OG } \\
\left(19.4-20.6{ }^{\circ} \mathrm{C}, 1 \mathrm{~d}\right) \\
\text { ㅇ 2nd call, } \\
\text { ㅇ 2nd answer }\end{array}$} & 2 & 2 & & & & $4-6$ & $3-9$ & \\
\hline & $\overline{72}$ & $\overline{72}$ & & & & $5.2 \pm 0.5$ & $\overline{5.5 \pm 1.6}$ & \\
\hline & 2 & 2 & & & & 3-8 & $1-5$ & \\
\hline & $\overline{72}$ & $\overline{56}$ & & & & $\overline{6.0 \pm 1.1}$ & $\overline{2.2 \pm 0.8}$ & \\
\hline \multirow{2}{*}{$\begin{array}{l}\text { C. californica, BCC } \\
\qquad\left(20.8^{\circ} \mathrm{C}, 1-7 \mathrm{~d}\right)\end{array}$} & 4 & 1 & & & & $1^{\mathrm{a}}$ & $1-4$ & \\
\hline & 102 & $\overline{47}$ & & & & $1.0 \pm 0.0^{\mathrm{a}}$ & $2.4 \pm 1.1$ & \\
\hline \multirow{4}{*}{ 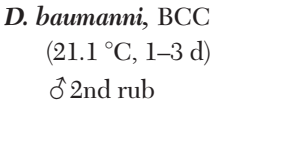 } & 3 & 1 & & & & $2^{\mathrm{a}}$ & 3-8 & \\
\hline & 60 & 60 & & & & $2.0 \pm 0.0^{\mathrm{a}}$ & $5.4 \pm 1.2$ & \\
\hline & 3 & & & & & & & \\
\hline & 60 & & & & & & & \\
\hline \multirow{2}{*}{$\begin{array}{l}\text { K. takhoma, CCT } \\
\left(21.5^{\circ} \mathrm{C}, 2 \mathrm{~d}\right)\end{array}$} & 1 & & & $4-5$ & $3-4$ & $12-13$ & & \\
\hline & 3 & & & $\overline{4.3 \pm 0.6}$ & $\overline{3.1 \pm 0.3}$ & $\overline{12.3 \pm 0.6}$ & & \\
\hline \multirow{2}{*}{$\begin{array}{l}\text { M. complicata, EWC } \\
\left(21.7^{\circ} \mathrm{C}, 8 \mathrm{~d}\right)\end{array}$} & 1 & & & $13-20$ & $1-3$ & $32-50$ & & \\
\hline & $\overline{27}$ & & & $\overline{17.5 \pm 1.8}$ & $\overline{2.8 \pm 0.5}$ & $\overline{40.1 \pm 4.5}$ & & \\
\hline \multirow{4}{*}{$\begin{array}{l}\text { N. spiniloba, CC\&T } \\
\text { VB }\left(20.5^{\circ} \mathrm{C}, 1-3 \mathrm{~d}\right) \\
\widehat{\delta}_{\mathrm{C}} \text { Grouped }\end{array}$} & 4 & 1 & 1 & & & $13-24$ & $5(n=1)$ & \\
\hline & $\overline{37}$ & $\overline{1}$ & $\overline{4}$ & & & $\overline{18.1 \pm 2.4}$ & & \\
\hline & 4 & & & $5-8$ & $3-5$ & 20-34 & & \\
\hline & $\overline{37}$ & & & $\overline{6.7 \pm 0.8}$ & $\overline{4.0 \pm 0.3}$ & $\overline{27.3 \pm 3.0}$ & & \\
\hline \multirow[t]{2}{*}{$\uparrow$ Grouped } & & 2 & & 5-15 & $1-5$ & & 18-61 & \\
\hline & & 11 & & $10.6 \pm 3.0$ & $3.8 \pm 0.5$ & & $42.7 \pm 14.9$ & \\
\hline \multirow[t]{2}{*}{$\sigma_{\mathrm{R}}$} & & & 1 & 19-22 & $1-6$ & & & 79-101 \\
\hline & & & $\overline{4}$ & $\overline{20.2 \pm 1.3}$ & $\overline{4.5 \pm 1.0}$ & & & $92.0 \pm 9.3$ \\
\hline \multirow{2}{*}{$\begin{array}{c}\text { O. vanduzeea, CC\&T } \\
\left(21.1^{\circ} \mathrm{C}, 1-2 \mathrm{~d}\right)\end{array}$} & 3 & 1 & & & & $6-11$ & $27-33$ & \\
\hline & 104 & 3 & & & & $9.1 \pm 1.0$ & $30.7 \pm 3.2$ & \\
\hline \multirow{2}{*}{$\begin{array}{l}\text { O. vanduzeea } \mathrm{OG} \\
\qquad\left(21.1-21.6^{\circ} \mathrm{C}, 1-5 \mathrm{~d}\right)\end{array}$} & 5 & & & & & $7-12$ & & \\
\hline & 137 & & & & & $10.3 \pm 0.9$ & & \\
\hline \multirow{2}{*}{$\begin{array}{l}\text { P. princeps } \mathrm{LBC} \\
\qquad\left(21.1^{\circ} \mathrm{C}, 2-6 \mathrm{~d}\right)\end{array}$} & 3 & & $\underline{1}$ & & & $4-9$ & & $6-7$ \\
\hline & 45 & & 3 & & & $7.1 \pm 1.1$ & & $6.3 \pm 0.6$ \\
\hline \multirow{3}{*}{$\begin{array}{c}\text { S. cora, BCCH } \delta \text { 1st } \\
\left(22.8^{\circ} \mathrm{C}, 1-4 \mathrm{~d}\right) \\
\text { ๙ } 2 \text { nd interval }\end{array}$} & 5 & 3 & 1 & & & 3 & 1 & 1 \\
\hline & 112 & $\overline{52}$ & $23^{*}$ & & & $\overline{3.0 \pm 0.0}$ & $\overline{1.0 \pm 0.0}$ & $\overline{1.0 \pm 0.0}$ \\
\hline & & & & & & & & \\
\hline
\end{tabular}

* One 3-way signal and $22 \delta_{\mathrm{C}^{-}}^{-\delta_{\mathrm{R}}}$ signals without + .

measurements, and in order to reduce the use of confusing jargon, 3 new "standards" or criteria are proposed additions to the monophasic signal (or pattern) definition. (1) No monophasic signal intervals measured using current methods (Sandberg and Stewart 2003, 2006) have a completely even interbeat interval pattern, and varied beat-interval patterns range from slightly to extremely variable. Therefore, a reasonable and logical limit to the variation of monophasic signals is set to a maximum mean monophasic interval difference of $10 \mathrm{~ms}$. Without this interval pattern variation limit, there are no clear methods in determining the difference between monophasic and slightly variable varied beatinterval patterns. (2) If the majority of signals share a consistent interval pattern of increasing or decreasing intervals with less than a 10ms interval difference, then they should be interpreted as varied beat-interval signals. (3) Omit intervals with one observation from the mean interval pattern description because they may not represent a typical signal. More work with this species and others is needed to further test the proposed monophasic definition suggested here. The 10-ms maximum mean 
answer $(q)$, male response $\left(\hat{\sigma}_{R}\right)$, male first call $\left(\hat{\delta}_{1}\right)$, female first answer $\left(q_{1}\right)$, and varied beat-interval combination indicated by superscripts a-d, respectively. Gray cells indicate unique second male call $\left(\hat{o}_{2}\right)$ or female answer $\left(\phi_{2}\right)$

\begin{tabular}{|c|c|c|c|c|c|}
\hline \multirow[b]{2}{*}{$\begin{array}{l}\text { Species, water body } \\
\text { (Temperature, age) }\end{array}$} & \multicolumn{3}{|c|}{$\begin{array}{l}\text { Interbeat, intragroup }^{\mathbf{b}} \\
\text { intervals or rub duration } \mathbf{c}(\mathrm{ms}) \\
\quad([\text { Avg } \pm \mathrm{SD}] / \text { Range })\end{array}$} & \multirow{2}{*}{$\begin{array}{c}\begin{array}{c}\text { Intergroup } / \text { rubd } \\
\text { intervals }(\mathrm{ms})\end{array} \\
\frac{([\mathrm{Avg} \pm \mathrm{SD}] / \text { Range })}{\delta-\widehat{o}}\end{array}$} & \multirow{2}{*}{$\begin{array}{c}\text { Exchange } \\
\text { Intervals }(\mathrm{ms}) \\
([\text { Avg } \pm \mathrm{SD}] / \text { Range }) \\
\hat{\delta}_{\mathrm{C}}^{-q} \text { or } \\
\hat{\delta}_{1}^{-q_{1}}\end{array}$} \\
\hline & $\begin{array}{c}\hat{\sigma}_{\mathrm{C}} / \hat{\sigma}_{1} \\
\hat{\sigma}_{2}\end{array}$ & $\begin{array}{l}q_{/} q_{1} \\
q_{2}\end{array}$ & $\sigma_{R}$ & & \\
\hline \multirow{4}{*}{$\begin{array}{l}\text { B. maculata, OG } \\
\left(19.4-20.6{ }^{\circ} \mathrm{C}, 1 \mathrm{~d}\right) \\
\text { ㅇ 2nd call, } \\
\text { ㅇ 2nd answer }\end{array}$} & $32.6 \pm 1.6$ & $54.2 \pm 14.3$ & & & $292.0 \pm 26.2$ \\
\hline & $\overline{27.9-38.6}$ & $\overline{35.3-106.4}$ & & & $\overline{233.6-413.2}$ \\
\hline & $\underline{49.8 \pm 8.4}$ & $72.9 \pm 15.8$ & & & \\
\hline & $\overline{37.9-93.4}$ & $43.5-120.9$ & & & \\
\hline C. californica, BCC & $89.4 \pm 18.7^{\mathrm{c}}$ & $144.7 \pm 35.7$ & & & $\underline{452.6 \pm 107.7}$ \\
\hline$\left(20.8^{\circ} \mathrm{C}, 1-7 \mathrm{~d}\right)$ & $44.8-163.3^{\mathrm{c}}$ & 83.8-259.6 & & & 281.7-680.1 \\
\hline D. baumanni, BCC & $\underline{57.8 \pm 8.8^{\mathbf{c}}}$ & $\underline{151.0 \pm 19.0}$ & & $\underline{144.5 \pm 16.0^{\mathrm{d}}}$ & $\underline{163.4 \pm 41.1}$ \\
\hline$\left(21.1^{\circ} \mathrm{C}, 1-3 \mathrm{~d}\right)$ & $\overline{41.1-83.8^{\mathrm{c}}}$ & $\overline{117.7-206.2}$ & & $\overline{108.2-181.3^{\mathrm{d}}}$ & $\overline{77.2-263.2}$ \\
\hline ô2nd rub & $\frac{117.7 \pm 16.6^{\mathbf{c}}}{80.3-154.3^{c}}$ & & & & \\
\hline $\begin{array}{l}\text { K. takhoma, CCT } \\
\left(21.5^{\circ} \mathrm{C}, 2 \mathrm{~d}\right)\end{array}$ & $\frac{66.9 \pm 3.0^{\mathbf{b}}}{59.9-73.2^{\mathbf{b}}}$ & & & $\frac{1242.0 \pm 163.2}{1082-1537}$ & \\
\hline $\begin{array}{l}\text { M. complicata, EWC } \\
\quad\left(21.7^{\circ} \mathrm{C}, 8 \mathrm{~d}\right)\end{array}$ & $\frac{25.64 \pm 2.7^{\mathbf{b}}}{18.8-35.8^{\mathbf{b}}}$ & & & $\frac{318.0 \pm 11.7}{246.3-368.5}$ & \\
\hline $\begin{array}{l}\text { N. spiniloba, CC\&T } \\
\quad \operatorname{VB}\left(20.5^{\circ} \mathrm{C}, 1-3 \mathrm{~d}\right)\end{array}$ & $\frac{74.6 \pm 43.2}{19.0-166.0}$ & $\frac{152.6 \pm 19.7}{124.2-167.0}$ & & & $703.4(n=1)$ \\
\hline$\hat{\sigma}_{\mathrm{C}}$ Grouped & $\frac{22.6 \pm 2.5^{\mathbf{b}}}{16.0-37.5^{\mathbf{b}}}$ & & & $\frac{204.6 \pm 13.9}{169.5-267.7}$ & \\
\hline q Grouped & & $\frac{24.5 \pm 2.5^{\mathbf{b}}}{17.8-43.3^{\mathbf{b}}}$ & & $\frac{191.1 \pm 39.4}{131.8-429.7}$ & \\
\hline$\delta_{R}$ & & & $\frac{23.5 \pm 3.1^{\mathbf{b}}}{18.6-42.7^{\mathbf{b}}}$ & $\frac{176.5 \pm 42.4}{60.4-297.5}$ & \\
\hline $\begin{array}{l}\text { O. vanduzeea, CC\&T } \\
\left(21.1^{\circ} \mathrm{C}, 1-2 \mathrm{~d}\right)\end{array}$ & $\frac{20.3 \pm 2.5}{14.3-25.2}$ & $\frac{22.3 \pm 1.2}{21.3-28.5}$ & & & $\frac{159.9 \pm 38.4}{118.3-194.1}$ \\
\hline $\begin{array}{l}\text { O. vanduzeea } \mathrm{OG} \\
\left(21.1-21.6^{\circ} \mathrm{C}, 1-5 \mathrm{~d}\right)\end{array}$ & $\frac{22.8 \pm 1.3}{19.6-30.0}$ & & & & \\
\hline $\begin{array}{l}\text { P. princeps } \mathrm{LBC} \\
\qquad\left(21.1^{\circ} \mathrm{C}, 2-6 \mathrm{~d}\right)\end{array}$ & $\frac{359.4 \pm 48.0}{212.8-488.4}$ & & $\frac{289.7 \pm 33.4}{244.0-372.6}$ & & \\
\hline $\begin{array}{l}\text { S. cora, BCCH } \delta \text { lst } \\
\left(22.8^{\circ} \mathrm{C}, 1-4 \mathrm{~d}\right) \\
\text { oै } 2 \text { nd interval }\end{array}$ & $\begin{array}{c}\frac{201.2 \pm 19.0}{151.5-238.6} \\
\frac{87.9 \pm 9.3}{67.6-108.9}\end{array}$ & & & & $\frac{619.4 \pm 103.1}{334.7-818.3}$ \\
\hline
\end{tabular}

monophasic interval difference was determined empirically by the author from observing drumming variation in this and other species.

Stewart et al. (1991) first described drumming in this species from 4 northern California foothill locations (Tables 1, 3). They recorded 4 males (at somewhat similar temperatures and unknown ages) with a range of 4-5 beats per call and intervals with a range of $29.7-33.3 \mathrm{~ms}$; however, 2 of their males had distinctly shorter average intervals than reported here. They suggested that the Napa and El Dorado county males $(n=2)$ with shorter intervals possibly represent a drumming dialect within this species (last 2 entries in Table 3).

This report is the first description for a 4-way exchange composed of 2 distinct call and answer signals in Plecoptera. Additional new signal and exchange types from other species will likely be detected when individual mean interval patterns are analyzed instead of a single overall average interval. The drumming of B. maculata requires further experimental study to better describe the typical signals through careful rearing of a population subsample and recording at known ages with controlled environmental 
TABLE 3. Detailed signal characters from 8 published descriptions of stonefly species and congener Kathroperla perdita at least some answers overlapped (O). Total number of male rubs, intergroup interval (ms), rub duration (ms), and inter-rub

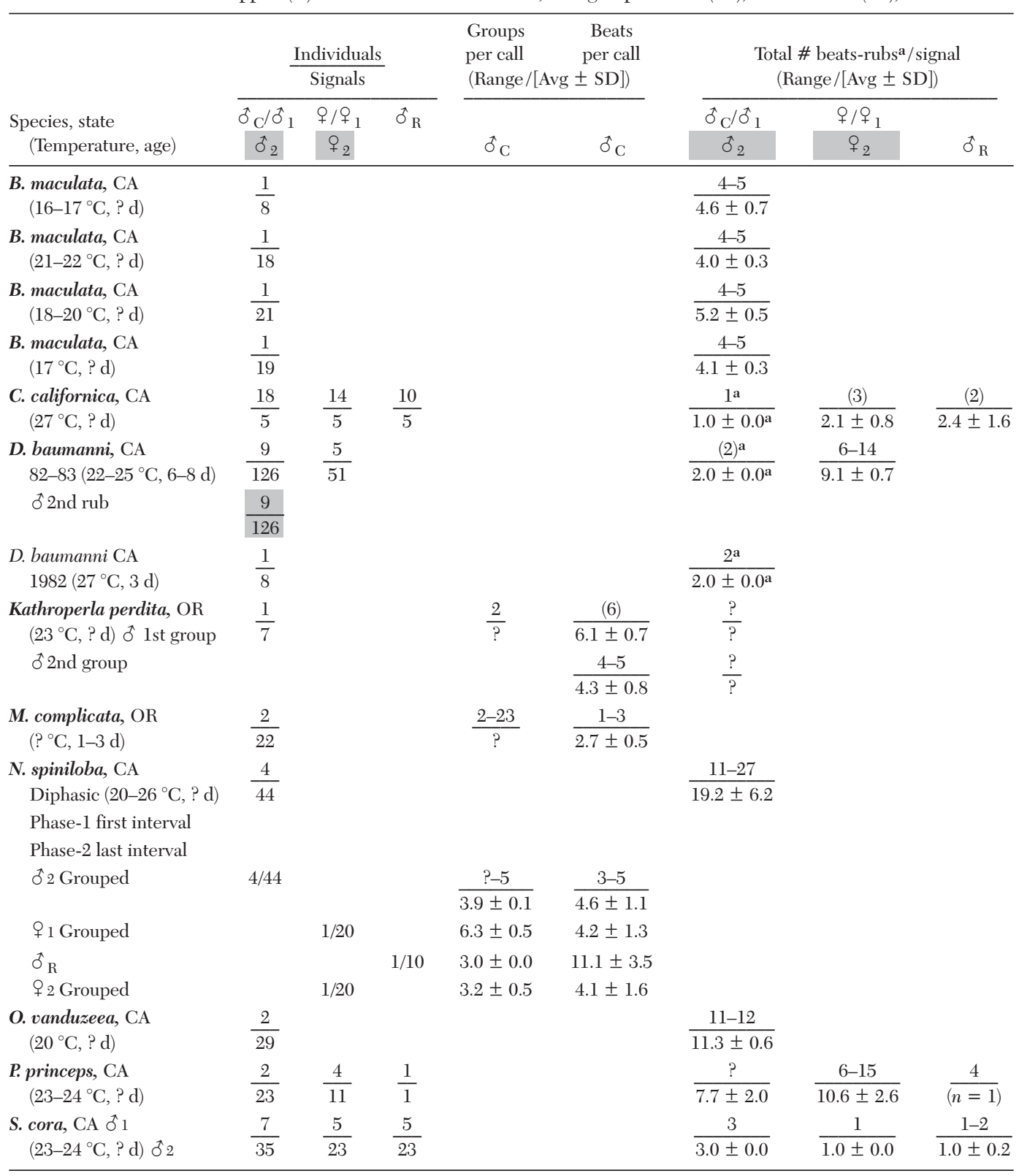

variables. Then, proposed species dialects from different populations could be supported by statistical analysis.

\section{CHLOROPERLIDAE}

KaTHROPERLA TAKHOMA.-This is the first drumming description for this species; however, so few signals were recorded that this description is considered preliminary. Three call signals were recorded at $21.5{ }^{\circ} \mathrm{C}$ from one $2 \mathrm{~d}$ male collected wild and teneral. The male called with grouped signals containing $4.3 \pm 0.6$ groups per signal (4-5), $3.1 \pm 0.3$ monophasic beats per group (3-4), and a total of $12-13$ beats per signal (Fig. 2, Table 2). Intragroup and intergroup intervals were $66.9 \pm 3.0 \mathrm{~ms}$ and $1242.0 \pm 163.2$ $\mathrm{ms}$, respectively. Call durations were variable $(4741.9 \pm 879.2 \mathrm{~ms})$ and depended upon the number of beats per signal. 
(Table 1). Male call $\left(\widehat{\delta}_{\mathrm{C}}\right)$, female answer $(q)$, male response $\left(\widehat{\delta}_{\mathrm{R}}\right)$, mode (number in parentheses), data not available (?), and interval (ms) are indicated by superscripts a-d, respectively. Gray cells indicate unique second male call signal characters.

\begin{tabular}{|c|c|c|c|c|c|}
\hline \multirow[b]{2}{*}{$\begin{array}{l}\text { Species, state } \\
\quad \text { (Temperature, age) }\end{array}$} & \multicolumn{3}{|c|}{$\begin{array}{l}\text { Interbeat, intergroup } \mathbf{b} \\
\text { intervals or rub duration }{ }^{\mathbf{c}}(\mathrm{ms}) \\
\quad([\text { Avg } \pm \mathrm{SD}] / \text { Range })\end{array}$} & \multirow{2}{*}{$\begin{array}{c}\begin{array}{c}\text { Intergroup } / \text { rub }^{\mathbf{d}} \\
\text { intervals (ms) } \\
([\text { Avg } \pm \mathrm{SD}] / \text { Range })\end{array} \\
\frac{\delta-\hat{\sigma}}{}\end{array}$} & \multirow{2}{*}{$\begin{array}{c}\begin{array}{c}\text { Exchange } \\
\text { intervals }(\mathrm{ms}) \\
([\text { Avg } \pm \mathrm{SD}] / \text { Range })\end{array} \\
\hat{\delta}_{\mathrm{C}^{-}+} \text {or } \\
\hat{\delta}_{1^{-+}}\end{array}$} \\
\hline & $\begin{array}{c}\hat{O}_{\mathrm{C}} / \hat{\delta}_{1} \\
\hat{\sigma}_{2}\end{array}$ & $\begin{array}{l}q_{/} q_{1} \\
q_{2}\end{array}$ & $\hat{o}_{R}$ & & \\
\hline $\begin{array}{l}\text { B. maculata, CA } \\
\left(16-17^{\circ} \mathrm{C}, ? \mathrm{~d}\right)\end{array}$ & $\frac{33.3 \pm 1.7}{?}$ & & & & \\
\hline $\begin{array}{l}\text { B. maculata, CA } \\
\left(21-22^{\circ} \mathrm{C}, ? \mathrm{~d}\right)\end{array}$ & $\frac{29.7 \pm 1.1}{?}$ & & & & \\
\hline $\begin{array}{l}\text { B. maculata, CA } \\
\left(18-20^{\circ} \mathrm{C}, ? \mathrm{~d}\right)\end{array}$ & $\frac{7.4 \pm 0.6}{?}$ & & & & \\
\hline $\begin{array}{l}\text { B. maculata, CA } \\
\left(17^{\circ} \mathrm{C}, ? \mathrm{~d}\right)\end{array}$ & $\frac{9.5 \pm 0.7}{?}$ & & & & \\
\hline $\begin{array}{l}\text { C. californica, CA } \\
\left(27^{\circ} \mathrm{C}, ? \mathrm{~d}\right)\end{array}$ & $\frac{\sim 70}{?}$ & $\frac{100.0 \pm 16}{?}$ & $\frac{100.0 \pm 26}{?}$ & & $\frac{?}{?}$ \\
\hline D. baumanni, CA & $66.2 \pm 15.0^{\mathrm{c}}$ & $\underline{129.6 \pm 5.0}$ & & $184.2 \pm 11.4^{\mathrm{d}}$ & $\underline{203.3 \pm 33.6}$ \\
\hline $\begin{array}{l}82-83\left(22-25^{\circ} \mathrm{C}, 6-8 \mathrm{~d}\right) \\
\text { § } 2 \text { nd rub }\end{array}$ & $\begin{array}{c}? \\
\frac{96.7 \pm 12.8^{c}}{?}\end{array}$ & $?$ & & $\begin{array}{l}? \\
? \\
\frac{?}{?}\end{array}$ & $?$ \\
\hline $\begin{array}{l}\text { D. baumanni, CA } \\
1982\left(27^{\circ} \mathrm{C}, 3 \mathrm{~d}\right)\end{array}$ & $\frac{?}{?}$ & & & $\frac{150.3 \pm 20.6^{\mathrm{d}}}{?}$ & \\
\hline 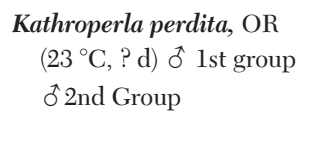 & $\begin{array}{c}\frac{41 \pm 6,34 \pm 7}{?} \\
\frac{23 \pm 2}{?}\end{array}$ & & & $\frac{139 \pm 20}{?}$ & \\
\hline $\begin{array}{l}\text { M. complicata, OR } \\
\left(?^{\circ} \mathrm{C}, 1-3 \mathrm{~d}\right)\end{array}$ & $\frac{30.3 \pm 4.4}{?}$ & & & $\frac{326.7 \pm 43.2}{234.8-452.7}$ & \\
\hline \multicolumn{6}{|l|}{$\begin{array}{l}\text { N. spiniloba, CA } \\
\quad \text { Diphasic }\left(20-26{ }^{\circ} \mathrm{C}, ? \mathrm{~d}\right)\end{array}$} \\
\hline Phase-1 first interval & $117.7 \pm ?$ & & & & \\
\hline Phase-2 last interval & $25.2 \pm ?$ & & & & \\
\hline$\delta_{2} 2$ Grouped & $\frac{7.3 \pm 1.2^{\mathbf{b}}}{?}$ & & & $\frac{58.6 \pm 8.7}{?}$ & \\
\hline $\begin{array}{l}q_{1 \text { Grouped }} \\
\hat{O}_{\mathrm{R}} \\
\Phi_{2 \text { Grouped }}\end{array}$ & $\begin{array}{c}6.1 \pm 0.8^{\mathbf{b}} \\
17.3 \pm 5.8^{\mathbf{b}} \\
8.5 \pm 1.6^{\mathbf{b}}\end{array}$ & & & $\begin{array}{c}54.4 \pm 16.1 \\
64.8 \pm 12.3 \\
81.2 \pm 4.6\end{array}$ & \\
\hline $\begin{array}{l}\text { O. vanduzeea, CA } \\
\left(20^{\circ} \mathrm{C}, ? \mathrm{~d}\right)\end{array}$ & $\frac{12.9 \pm 0.8}{?}$ & & & & \\
\hline $\begin{array}{l}\text { P. princeps, CA } \\
\left(23-24^{\circ} \mathrm{C}, ? \mathrm{~d}\right)\end{array}$ & $\frac{388 \pm 22}{?}$ & $\frac{385 \pm 26}{?}$ & $\frac{438 \pm 27}{435-478}$ & & $\frac{(\mathrm{O})-375}{?}$ \\
\hline $\begin{array}{l}\text { S. cora, CA ठै 1 } \\
\left(23-24^{\circ} \mathrm{C}, ? \mathrm{~d}\right) \delta_{2}\end{array}$ & $\frac{146 \pm 7}{61 \pm 12}$ & & $432(n=1)$ & & $\frac{373 \pm 30}{?}$ \\
\hline
\end{tabular}

The intragroup and intergroup interval call patterns increased (Figs. 7-8). The monophasic intragroup interval pattern increased slightly from $66.4 \mathrm{~ms}$ (i1) to $67.6 \mathrm{~ms}$ (i2). The last interval (i3) decreased to $63.8 \mathrm{~ms}(n=1)$. The intergroup interval pattern increased from 1111.4 $\mathrm{ms}$ (i1) to $1366.0 \mathrm{~ms}$ (i3), and interval 4 (i4) increased to $1474.9 \mathrm{~ms}(n=1)$.

Stewart and Zeigler (1984) described 7 call signals of congener Kathroperla perdita (Banks) as diphasic (Tables 1, 3). This species' call signal description is interpreted here as bi-grouped, as opposed to diphasic, because (1) no major continuous changes in beat interval occurred within the groups (Stewart and Sandberg 2006), (2) the variable call signals were repeated in grouped patterns (Stewart and Sandberg 2006), and (3) a possible answer signal could be placed between the call groups. There will always be uncertainty in signal descriptions lacking the 

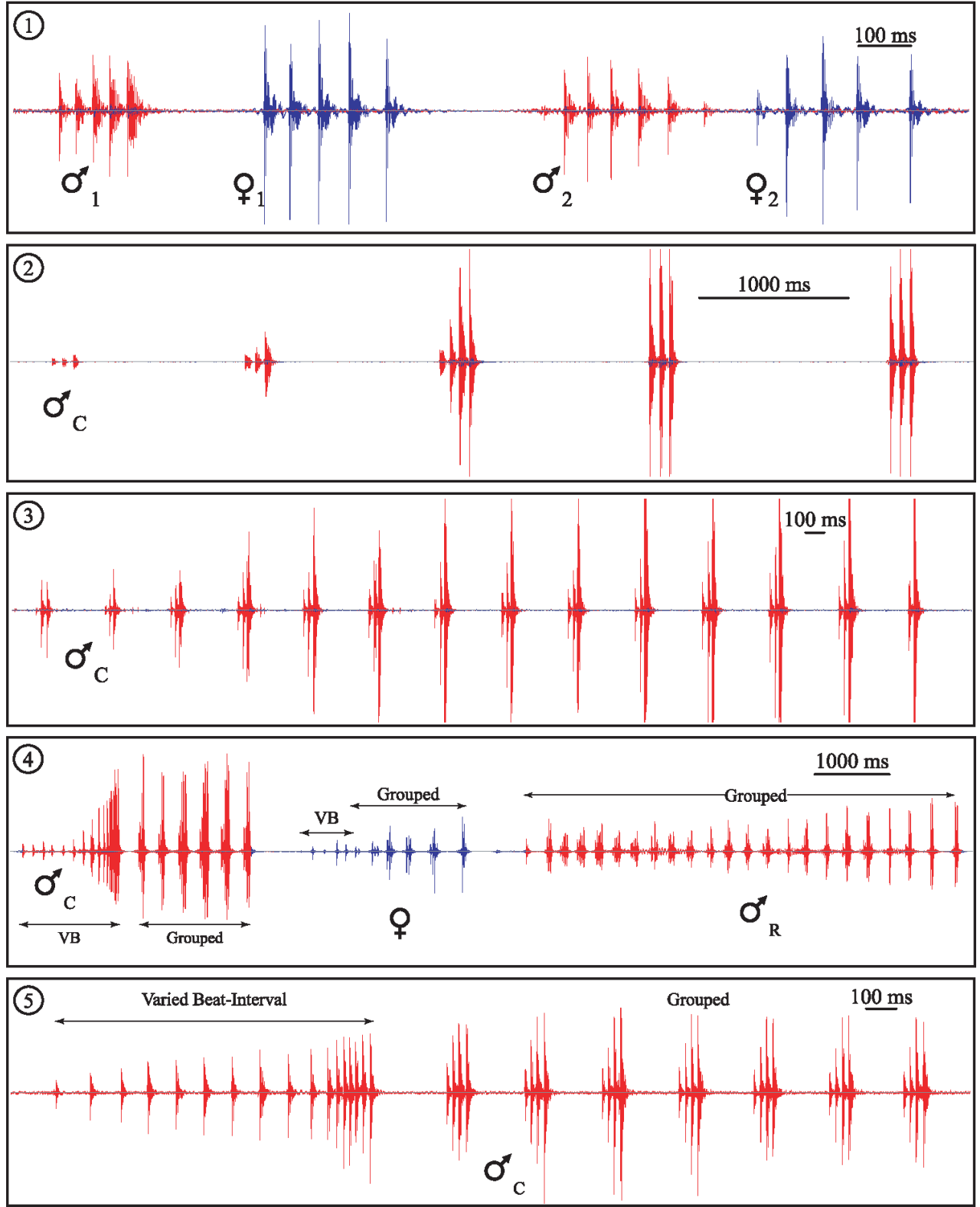

Figs. 1-5. Stonefly drumming signals: 1 , Bolshecapnia maculata 4 -way exchange with monophasic $\delta_{1}$, varied beatinterval $\hat{\delta}_{2}$, and varied beat-interval $\phi_{1^{-}} \phi_{2}$ signals; 2 , Kathroperla takhoma grouped call; 3, Megaleuctra complicata grouped (tribeat) call; 4, Nemoura spiniloba combination varied beat-interval (VB) and grouped call, combination varied beat-interval and grouped answer, and grouped response; 5, Nemoura spiniloba combination varied beat-interval and grouped call. Call $\left(\hat{\sigma}_{\mathrm{C}}\right)$, answer $(\hat{q})$, and response $\left(\hat{\delta}_{\mathrm{R}}\right)$.

female answer. In Stewart and Zeigler (1984), the first group's mean intragroup intervals were $36 \pm 5 \mathrm{~ms}$, and individual intervals decreased from $41 \mathrm{~ms}$ to $34 \mathrm{~ms}$. Also, the second group's intervals were relatively even at $23 \pm 2 \mathrm{~ms}$ (similar to Bolshecapnia maculata intervals above). These interval data fit the grouped signal definition (Stewart and Sandberg 2006) with percussive bursts, each of a few beats, and variable intragroup time intervals. The current report adds a second grouped call signal to the known drumming of Kathroperla. 

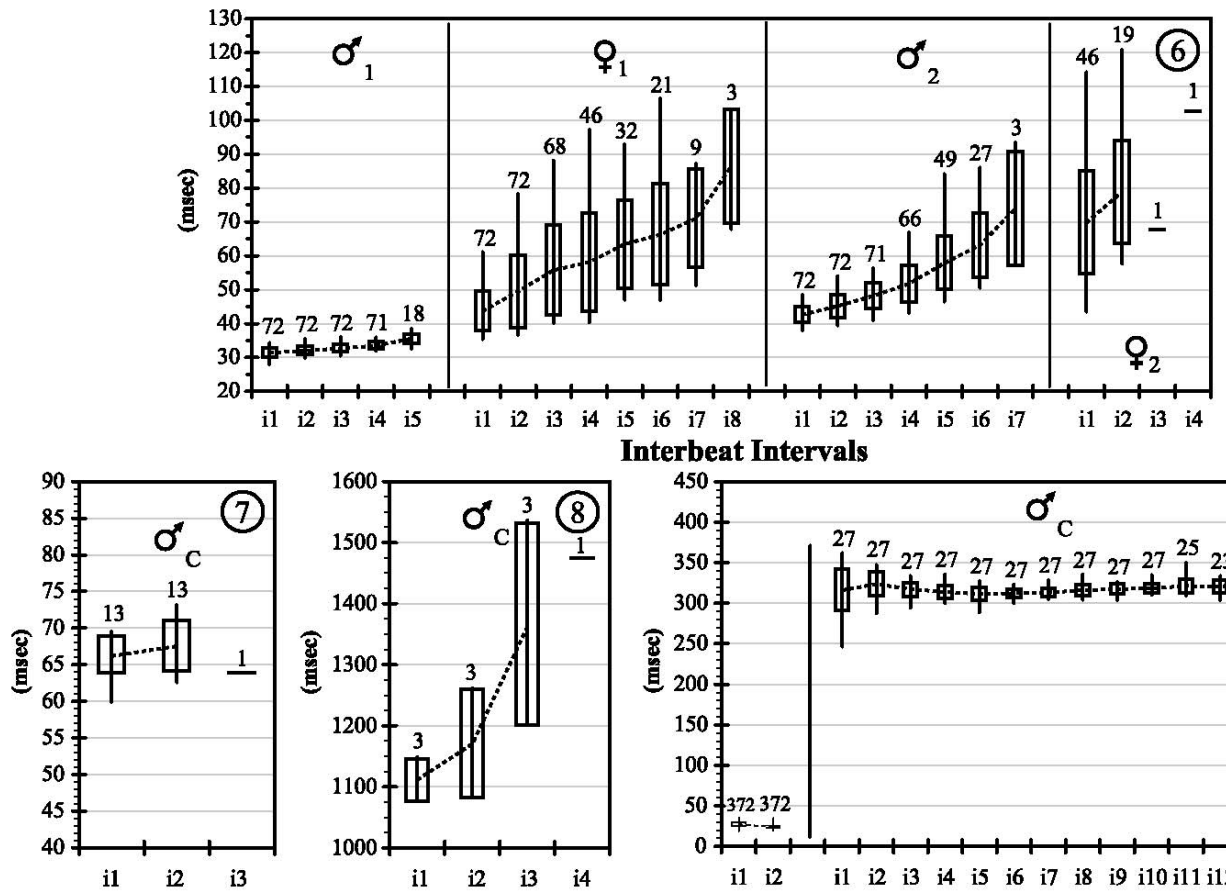

Intervals

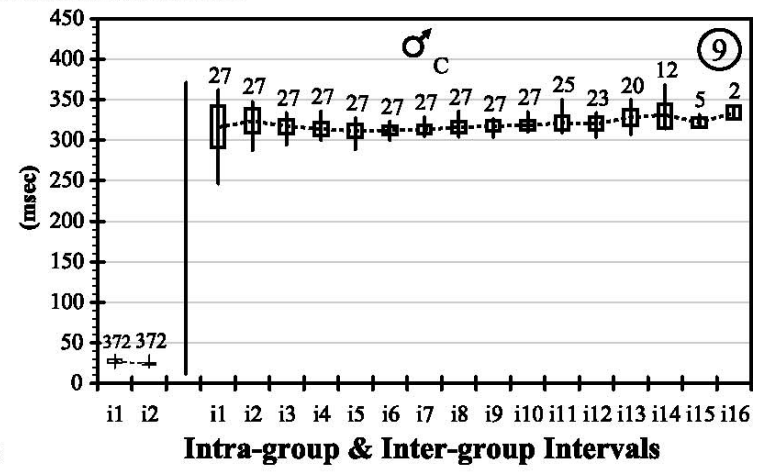

Intra-group Intervals Inter-group Intervals
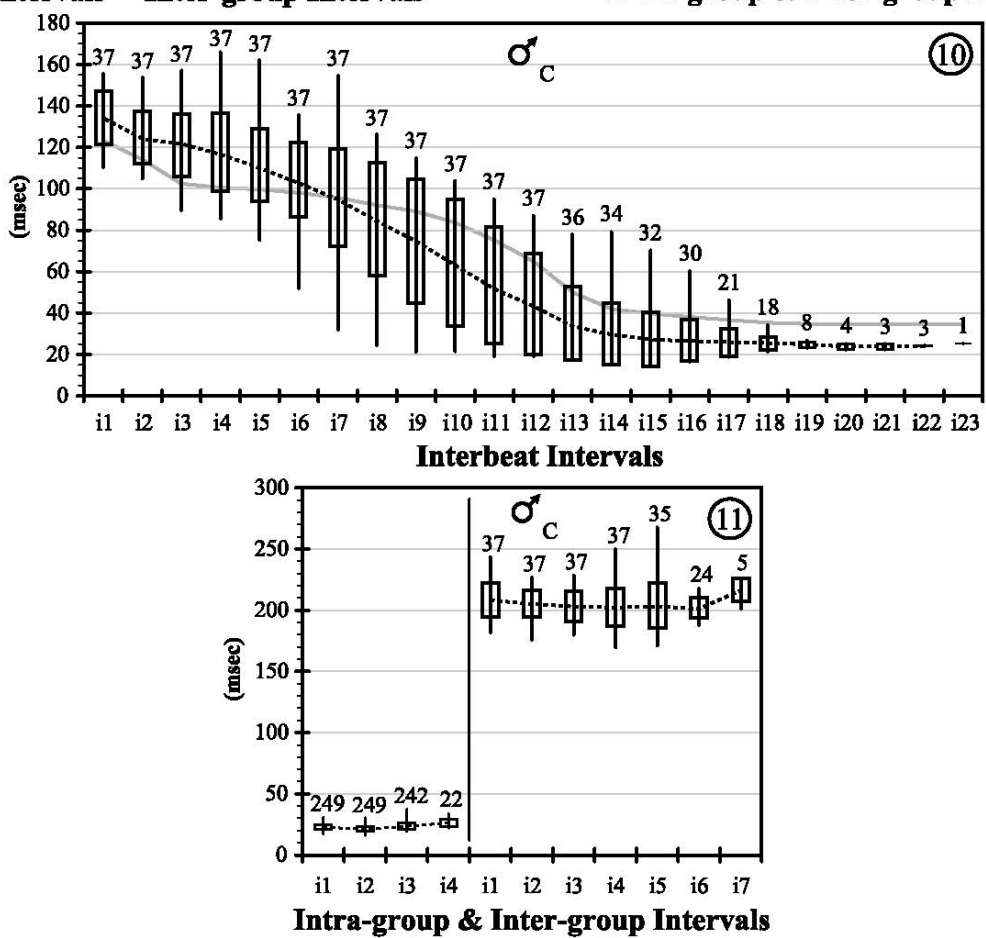

Figs. 6-11. Drumming signal interbeat, intragroup, and intergroup interval patterns: 6, Bolshecapnia maculata

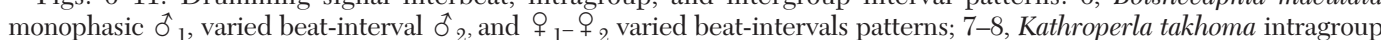
and intergroup patterns; 9, Megaleuctra complicata intragroup (left) and intergroup (right) patterns; 10-11, Nemoura spiniloba varied beat-interval pattern (10), intragroup pattern (11 left) and intergroup pattern (11 right). Mean $=$ dashed line, standard deviation $=$ box, range $=$ vertical line, number of intervals $=$ number above the range 

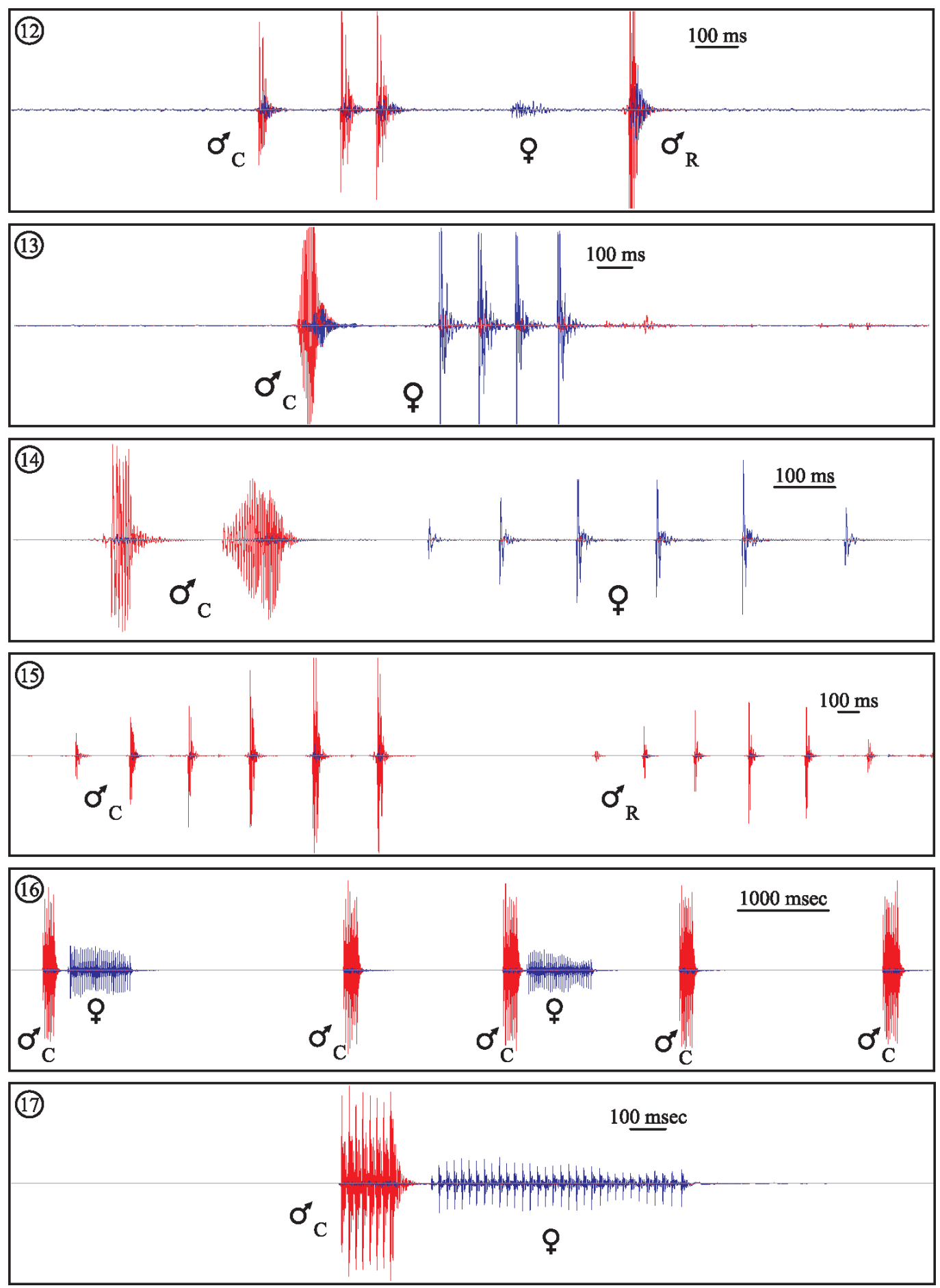

Figs. 12-17. Stonefly drumming signals: 12, Sierraperla cora varied beat-interval call with one-beat monophasic answer and response; 13, Calineuria californica rub call and varied beat-interval answer; 14, Doroneuria baumanni bi-rub call and varied beat-interval answer; 15, Pteronarcys princeps varied beat-interval call and response; 16, Oemopteryx vanduzeea repeated monophasic call and answer, CC\&T; 17, Oemopteryx vanduzeea horizontal zoom of $\hat{\delta}-q$ exchange from above. Call $\left(\hat{\delta}_{\mathrm{C}}\right)$, answer $(\hat{q})$, and response $\left(\hat{\delta}_{\mathrm{R}}\right)$. 

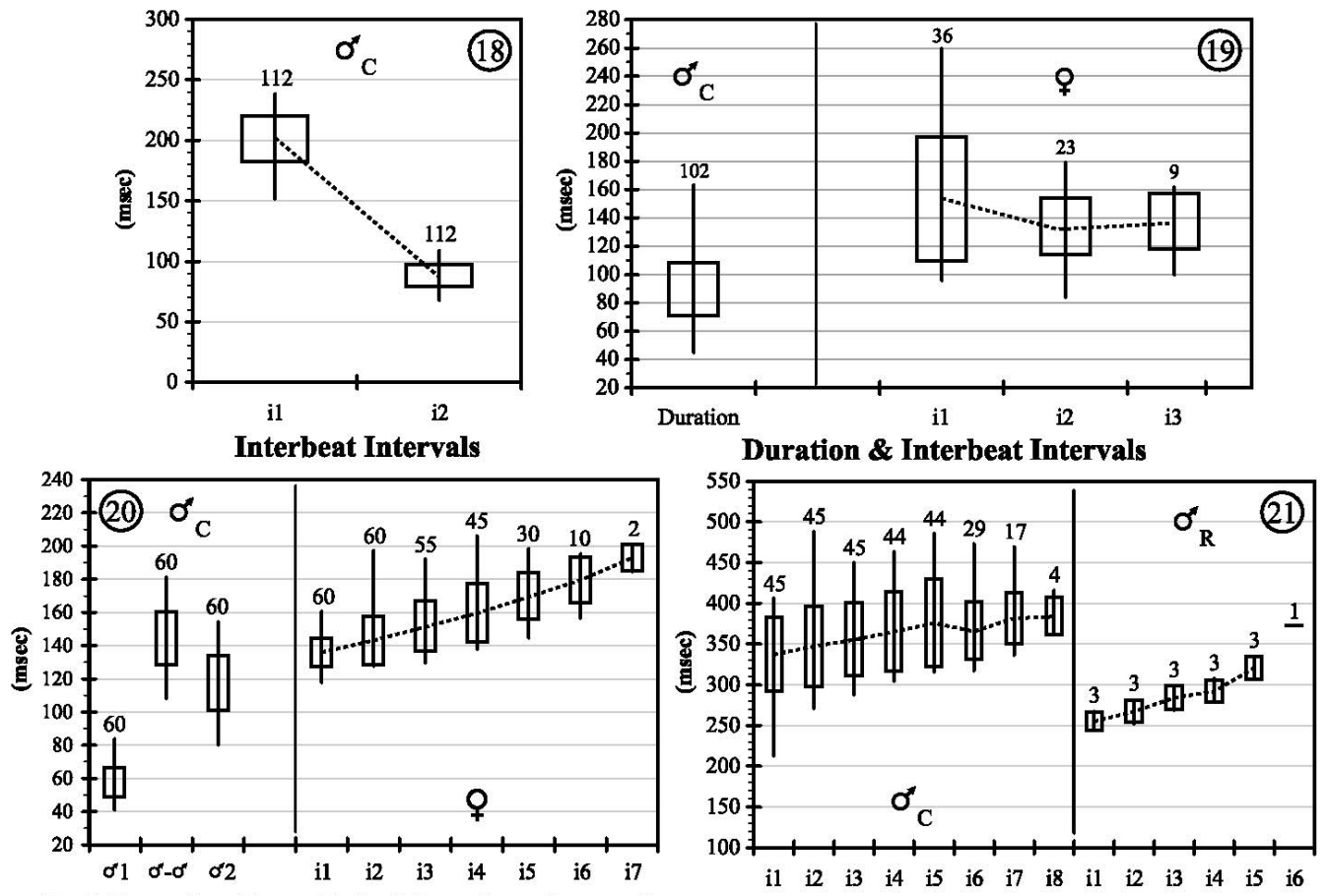

Rub Duration, Inter-Rub \& Interbeat Intervals
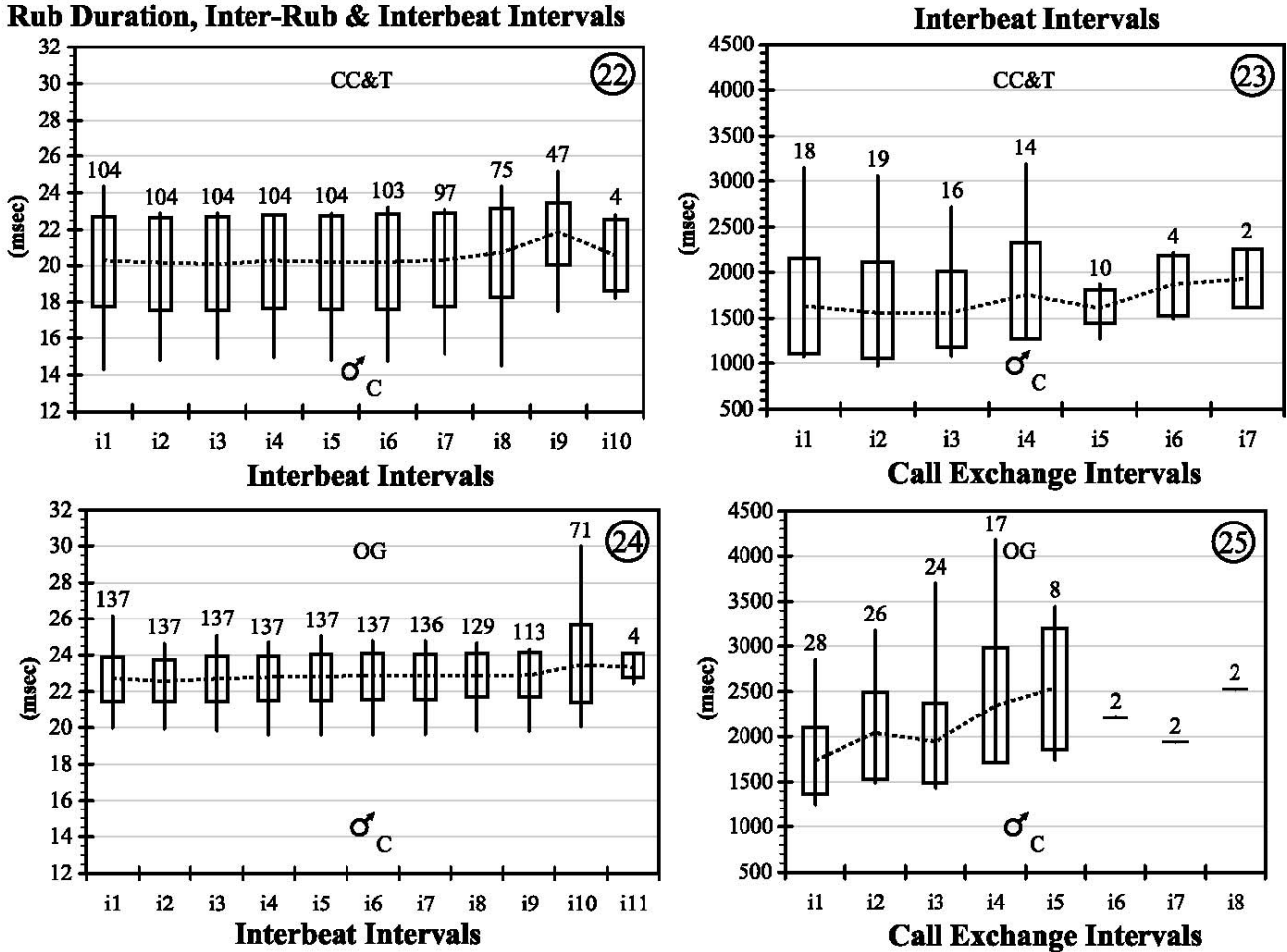

Figs. 18-25. Drumming signal rub duration, inter-rub, interbeat and repeated call exchange interval patterns: 18, Sierraperla cora varied beat-interval call pattern; 19, Calineuria californica rub call duration and varied beat-interval answer pattern; 20, Doroneuria baumanni bi-rub call and varied beat-interval answer; 21, Pteronarcys princeps varied beat-interval call and response; 22-25, Oemopteryx vanduzeea repeated monophasic call and exchange interval patterns. Mean $=$ dashed line, standard deviation $=$ box, range $=$ vertical line, number of intervals $=$ number above the range. 


\section{LEUCTRIDAE}

MEGALEUCTRA COMPLICATA.-Twenty-seven grouped call signals were recorded from one $8 \mathrm{~d}$ male at $21.7^{\circ} \mathrm{C}$. Males called with $17.5 \pm 1.8$ groups per signal (13-20), $2.8 \pm 0.5$ monophasic beats per group (1-3), and a total of 32-50 beats per signal (Fig. 3, Table 2). The average intragroup and intergroup intervals were $25.6 \pm 2.7 \mathrm{~ms}$ and $318.0 \pm 11.7 \mathrm{~ms}$, respectively. Call durations were $4834.1 \pm 675.64 \mathrm{~ms}$ (3322.5-6009.8). The monophasic intragroup interval pattern decreased slightly from $27.0 \mathrm{~ms}$ (il) to $24.0 \mathrm{~ms}$ (i2) (Fig. 9, left side), and the intergroup interval pattern increased slightly and irregularly (Fig. 9, right side). The intergroup pattern increased from $314.9 \mathrm{~ms}$ (i1) to $323.9 \mathrm{~ms}$ (i2), then decreased to a minimum of $311.0 \mathrm{~ms}$ (i5), and finally increased irregularly to a maximum of $334.0 \mathrm{~ms}$ (i16).

Stewart and Sandberg (2004) first reported this species drumming from two 1-3 d (or more) field-collected males that also produced grouped tri-beat calls (Table 3). Their average intragroup and intergroup intervals were slightly higher than those reported here, which may indicate the actual ages were greater, perhaps more than the $8 \mathrm{~d}$ individuals reported here. Increased age has been suspected to increase interbeat intervals (Sandberg and Stewart 2005). In Leuctridae, only the drumming of M. complicata and 4 Zealuectra Ricker species are known.

\section{NEMOURIDAE}

NEMOURA SPINILOBA. - Thirty-seven calls from 4 males, 12 answers from 2 females, and 4 response signals from one male were recorded at $20.5^{\circ} \mathrm{C}$ (age range $1-3 \mathrm{~d}$ ). The male call was complex, beginning with a varied beat-interval signal followed by a series of grouped signals interpreted here as a combination signal type: varied beat-interval and grouped (Sandberg 2009). The female answer for one exchange was a combination of several varied beat-interval beats followed by a short series of grouped signals (typical answer type), and the final male response signal was grouped (Table 1, Fig. 4). Almost all exchanges were 2-way $\left(\hat{o}_{\mathrm{C}^{-}}+\right)$and only one was 3-way $\left(\hat{o}_{\mathrm{C}^{-}}+\underline{q}-\hat{0} \mathrm{R}\right)$

The initial varied beat-interval combination call component was composed of $18.1 \pm 2.4$ beats per signal (13-24) with $74.6 \pm 43.2 \mathrm{~ms}$ intervals (Fig. 5, Table 2). Varied beat-interval components had durations of $1277.6 \pm 229.1 \mathrm{~ms}$
(753.8-1660.9). In the following grouped component, males called with $6.7 \pm 0.8$ groups per signal (5-8), $4.0 \pm 0.3$ monophasic beats per group (3-5), with a total of 20-34 beats per grouped signal component (Fig. 5, Table 2). Intragroup and intergroup intervals were 22.6 $\pm 2.5 \mathrm{~ms}$ and $204.6 \pm 13.9 \mathrm{~ms}$, respectively.

The initial varied beat-interval call pattern was a decreasing sigmoid curve (Fig. 10). The pattern decreased from a maximum of $134.2 \mathrm{~ms}$ (i1) to $30.0 \mathrm{~ms}$ (i14), then became nearly even over intervals (i15-i22), decreasing slightly from $27.2 \mathrm{~ms}$ (i15) to a minimum of $23.7 \mathrm{~ms}$ (i20), and finally exhibiting a slight increase to $25.4 \mathrm{~ms}$ (i23) $(n=1)$. The intragroup and intergroup call patterns were approximately even (Fig. 11). The intragroup pattern (Fig. 11, left side) decreased slightly from $22.8 \mathrm{~ms}$ (i1) to a minimum of 21.1 $\mathrm{ms}$ (i2), then increased to a maximum of $26.2 \mathrm{~ms}$ (i4). The intergroup pattern (Fig. 11, right side) decreased irregularly from $208.3 \mathrm{~ms}$ (il) to a minimum of $201.8 \mathrm{~ms}$ (i6), then increased to $216.5 \mathrm{~ms}$ (i7).

One female answered a combination varied beat-interval and grouped male call in the recording chamber. The atypical answer began with 5 varied beat-interval beats with long intervals $152.6 \pm 19.7 \mathrm{~ms}$ (124.2-167.0), which were followed by the typical grouped signals also observed from the second female, who only answered the playback of calls from previously recorded males $(n=10)$. Because the atypical combination varied beat-interval and grouped answer was only observed once, it was omitted from the general description (Table 1). The female grouped answers had 5-15 groups per signal, 1-5 varied beat-interval beats per group, and a total of 18-61 beats per signal (Fig. 4, Table 2). The intragroup and intergroup intervals were $24.5 \pm 2.5 \mathrm{~ms}$ and $191.1 \pm 39.4 \mathrm{~ms}$, respectively. Answer durations were $2717.6 \pm$ $855.5 \mathrm{~ms}$ (1228.2-3856.1). The grouped male response $(n=4)$ had 19-22 groups per signal, 1-6 monophasic beats per group, and a total of 79-101 beats per signal (Fig. 4, Table 2). Intragroup and intergroup intervals were $23.5 \pm$ $3.1 \mathrm{~ms}$ and $176.5 \pm 42.4 \mathrm{~ms}$, respectively. For only one 3 -way exchange, the $\hat{o}_{\mathrm{C}^{-}}+9$ exchange interval was $703.4 \mathrm{~ms}$ (Table 2), and the $q_{-} \widehat{o}_{\mathrm{R}}$ exchange interval was $675.2 \mathrm{~ms}$.

The signal descriptions above are the second report of drumming for this species (Table 1). The original diphasic call description for 3 different California populations from El Dorado 
and Napa counties (Stewart et al. 1991) is updated here to the more complex combination signal type composed of varied beat-interval and grouped signal types. In Stewart et al. (1991), the term "diphasic" was used to describe 2 call components: a varied beat-interval "phase-1" signal, followed by a grouped "phase-2." The varied interbeat-interval and grouped call intervals from the results herein (Table 2) compared fairly well to the call intervals reported for the first and last diphasic intervals by Stewart et al. (1991) (Table 3). The grouped call portion from Stewart et al. (1991) compared less favorably to the results herein, with approximately half the mean number of groups per signal than reported here $(3.9 \pm 0.1$ vs. $6.7 \pm 0.8$, respectively), similar mean beats per group, and approximately $30 \%$ shorter intragroup and intergroup intervals (Tables 2-3). In Stewart et al. (1991), the answer and response, number of groups per signal, and beats per group were fewer than those reported here (Tables 2-3). Additionally, Stewart et al. (1991) reported a second female grouped answer $(n=20)$ that followed the male response (4-way exchange), which I did not observe.

The initial N. spiniloba varied beat-interval component of the combination call type (varied beat-interval and grouped) appeared similar to the entire diphasic call of Isoperla phalerata Needham (Szczytko and Stewart 1979, Sandberg and Stewart 2006). The diphasic I. phalerata call (Sandberg and Stewart 2006) was a series of up to 37 beats that began with variable but relatively even intervals. These intervals were followed by an interval decrease (or transition), and finally they returned to approximately even intervals. In signal-type terminology, there was a series of 3 successive and continuous call types: monophasic, varied beat-interval, and monophasic. The gray superimposed curved line in Fig. 10 is the I. phalerata interval pattern over intervals 1-23. And although the first 3 mean intervals decreased $20 \mathrm{~ms}$, there was an approximately even series of intervals over i4-i9, with only a slight decrease from $100 \mathrm{~ms}$ to 92 $\mathrm{ms}$. The $N$. spiniloba interval pattern decreased steadily over the initial half of the signal, then decreased its rate of change until becoming approximately even over the last intervals (Fig. 10, dashed black line). Because this pattern lacked the initial, nearly even intervals observed in I. phalerata, it is interpreted here as a varied beat-interval signal type. The diphasic signal type will require additional details and further study to make its definition distinct from varied beatinterval signals.

\section{PELTOPERLIDAE}

SIERRAPERLA CORA.-One hundred twelve calls from 5 males, 52 answers from 3 females, and 23 response signals from one male were recorded at $22.8^{\circ} \mathrm{C}$ (age range $1-4 \mathrm{~d}$ ). Males and females signaled with varied beat-interval calls, monophasic 1-beat answers, and 1-beat responses with 2-way $(n=26)$ and 3-way $(n=1)$ exchanges. Twenty-two male signals included both the male call and response but lacked the female answer. This observation was atypical because there were no females within the recording chamber or nearby to answer the calls. The male call always contained 3 beats with $201.2 \pm 19.0 \mathrm{~ms}$ first intervals and $87.9 \pm 9.3 \mathrm{~ms}$ second intervals (Fig. 12, Table 2). Call durations were short $289.1 \pm 28.0 \mathrm{~ms}(221.1-342.9)$. The

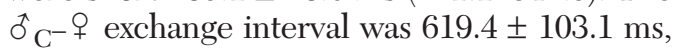
and the $q_{-} \widehat{O}_{\mathrm{R}}$ exchange interval was $291.9 \mathrm{~ms}$ $(n=1)$. Two females answered the playback of recorded male calls $(n=26)$.

The $S$. cora 3-beat interbeat interval call pattern decreased from $201.2 \mathrm{~ms}$ (il) to $87.9 \mathrm{~ms}$ (i2) (Fig. 18), with an interval difference of 113.3 ms. This pattern is similar to the newly discovered, second observed call type of Osobenus yakimae (Hoppe), a perlodid stonefly included in a concurrent study by the author. The O. yakimae calls recorded in 2010 had 3-beat varied beat-intervals which decreased from $132.3 \pm$ $6.4 \mathrm{~ms}$ to $70.2 \pm 4.4 \mathrm{~ms}$. Another 3-beat varied beat-interval call pattern for Perlinella drymo (Newman) was reported by Sandberg and Stewart (2006), Stewart et al. (1982), and Zeigler and Stewart (1977). Sandberg and Stewart (2006) reported a decreasing call pattern for $P$. drymo, from $92.3 \mathrm{~ms}$ (i1) to $26.9 \mathrm{~ms}$ (i2), and a 1-beat female answer followed the last call beat by $138.0 \pm 5.1 \mathrm{~ms}$.

This is the second report of drumming for S. cora. Zeigler and Stewart (1985) first reported the call, answer, and response signals for this species as monophasic (Table 1). The call description is updated here to varied beat-interval. Zeigler and Stewart (1985) also reported that exchanges ranged from 3-way to 5 -way, with a second female answer and male response signal from one of their test pairs. Their results differed slightly from this study, with 1-2 response beats and shorter first call intervals of $146.0 \pm 7.0 \mathrm{~ms}$ 
(Table 3). Also from their study, the $\hat{o}_{\mathrm{C}^{-}}$? exchange interval was within the range reported here, and their $q_{-} \hat{o}_{\mathrm{R}}$ exchange interval was longer at $383 \pm 35 \mathrm{~ms}$. They did not provide ages for their stoneflies, and perhaps their males were older than those reported here, which could account for their longer first-call intervals. Their second interval agreed well with this study. Sandberg and Stewart (2005) described the drumming of 2 Isogenoides elongatus (Hagen) Colorado River populations. The 10 fieldcollected adults from near Granby were assumed older than the 7 reared adults from near Rifle (no ages provided) because kicknet sampling for larvae was unsuccessful and no other adults were observed in usual habitats. The field-collected (assumed older) Granby population called with signals containing more beats on average and longer intervals than the reared Rifle population. Additional experimental studies with careful rearing and recording at known ages with controlled environmental variables will be required before the effects of age and temperature on drumming will be fully understood.

\section{PERLIDAE}

CALINEURIA CALIFORNICA. - One hundred two calls from 4 males and 47 answers from one female were recorded at $20.8^{\circ} \mathrm{C}$ (age range $1-7 \mathrm{~d}$ ). The 4 males called with one long rub signal, females answered with short varied beat-interval signals, and all exchanges were 2-way. The rub call duration was $89.4 \pm 18.7 \mathrm{~ms}$ (44.8-163.3; Fig. 13, Table 2). The number of beats per female answer signal was $2.4 \pm 1.1$ (1-4) with intervals of $144.7 \pm 35.7 \mathrm{~ms}$. Answer durations were $273.4 \pm 84.2 \mathrm{~ms}(95.7-430.4)$. The $\hat{o}^{-} \mathrm{C}^{-}$ㅇ exchange interval was $281.7-680.1 \mathrm{~ms}$, and 2way signal durations were $752.3 \pm 95.6 \mathrm{~ms}$ (472.1-901.53).

The rub call signal was not a series of individual percussive beats as in monophasic or diphasic signals (Stewart et al. 1982). Rub calls were produced by a continuous drag of the ventral abdomen, producing a nonpercussive squeak, and only its duration could be consistently measured and analyzed statistically. The rub duration was charted in Figure 19 (left side) with a mean of $89.4 \pm 18.7 \mathrm{~ms}$ (Table 2). The answer interval pattern decreased from a maximum of $153.5 \mathrm{~ms}$ (i1) to a minimum of $134.0 \mathrm{~ms}$ (i2), and then increased slightly to $137.5 \mathrm{~ms}$ (i3) (Fig. 19, right side).
The drumming for this species from Sierra County, Little Truckee River, was first reported by Stewart et al. (1982). They reported 3-way exchanges with monophasic answers and male response signals (Table 2). The rub duration and varied beat-interval answer intervals for the Butte County individuals from the current study (Table 2) were longer than those reported by Stewart et al. (Table 3), and were recorded up to 7 days after field collection. Increased age could account for the longer intervals and the absence of the male response signal for signals reported here (Sandberg and Stewart 2005).

DORONEURIA BAUMANNI._-Sixty calls from 3 males and 60 answers from one female were recorded at $21.1{ }^{\circ} \mathrm{C}$ (age range $1-3 \mathrm{~d}$ ). The males signaled consistently with 2 rub calls, and females answered with 2-way exchanges consisting of varied beat-interval signals. The first rub duration was $57.8 \pm 8.8 \mathrm{~ms}$ and the second was $117.7 \pm 16.6 \mathrm{~ms}$, with an inter-rub interval of $144.5 \pm 16.0 \mathrm{~ms}$ (Fig. 14, Table 2). Bi-rub call durations were $175.5 \pm 19.6 \mathrm{~ms}$ (134.2-230.2). The $\hat{o}_{\mathrm{C}}-9$ exchange interval was $163.4 \pm 41.1$ ms. The number of beats per female answer signal was $5.4 \pm 1.2(3-8)$ with intervals of $151.0 \pm 19.0 \mathrm{~ms}$ (Fig. 14, Table 2). The answer durations were $151.0 \pm 19.0 \mathrm{~ms}$ (117.7-206.2).

The first rub duration $\left(\delta_{1}\right)$, inter-rub interval $(\hat{O}-\hat{O})$, and second rub duration $\left(\hat{O}_{2}\right)$ are charted in Fig. 20 (left side), indicating an increasing bi-rub duration pattern. The interbeat interval answer pattern increased from $136.0 \mathrm{~ms}$ (i1) to $192.9 \mathrm{~ms}$ (i7) (Fig. 20, right side). Maketon and Stewart (1984) provided the first description for this species from another California population (Table 1). The drumming characters reported here compared well with the first study (Tables 2-3). The overall characters from Maketon and Stewart (1984) were reported in 2 sections: (1) 40 calls from 9 males in 1982 and 86 calls from 3 males in 1983, and (2) 8 calls from one male in 1982. There were always 2 rub calls reported in both studies, but the number of answer beats was higher in Maketon and Stewart (1984) (Table 3). Individual rub durations ( $\hat{o}_{1}$ and $\hat{\partial}_{2}$ ) increased similarly, and the inter-rub interval standard deviation overlapped in both studies. The first study also reported a decrease in overall inter-rub interval for 8 males recorded at the slightly higher temperature of $27{ }^{\circ} \mathrm{C}$ versus $22-25^{\circ} \mathrm{C}$, which is expected, as temperature has been suspected to affect drumming characters (Sandberg and Stewart 2005). 


\section{PTERONARCYIDAE}

PTERONARCYS PRINCEPS. - Forty-five call signals and 3 response signals were recorded from three 2-6 d males at $21.1{ }^{\circ} \mathrm{C}$. Males called and responded (without female answers) with varied beat-interval signals. The males called with signals of $7.1 \pm 1.1$ beats (4-9) with intervals of $359.4 \pm 48.0 \mathrm{~ms}$ (Fig. 15, Table 2). Males produced 3 response signals, which had $6.3 \pm 0.6$ beats $(6-7)$ with intervals of $289.7 \pm$ $33.4 \mathrm{~ms}$. The call interval pattern increased irregularly from a minimum of $337.3 \mathrm{~ms}$ (i1) to a maximum of $384.4 \mathrm{~ms}$ (i8) (Fig. 21, left side). The suspected response pattern increased from a minimum of $255.3 \mathrm{~ms}$ (i1) to a maximum of $320.9 \mathrm{~ms}$ (i5) (Fig. 21, right side). The last interval increased to $372.6 \mathrm{~ms}(\mathrm{i} 6)(n=1)$.

This is the second report for this species (Table 1). Zeigler and Stewart (1985) first described monophasic calls and responses with overlapped monophasic answers, and 3-way exchanges for this species. Their overall characters compared well with the results herein except that response interbeat intervals were approximately $150 \mathrm{~ms}$ longer in their study (Table 3).

\section{TAENIOPTERYGIDAE}

OEMOPTERYX VANDUZEEA.-Two additional California populations located in intermittent streams of the Sierra Nevada (Campbell Creek and Oregon Gulch) and separated by less than 2 miles $(3.2 \mathrm{~km})$ are reported here. This is the second report for this species (Stewart et al. 1991), and the female answer is tentatively described from 3 signals (Table 1).

Campbell Creek and tributaries: One hundred four calls from three 1-2 d males and 3 answers from one $1 \mathrm{~d}$ female were recorded at $21.1^{\circ} \mathrm{C}$. The males signaled with widely and irregularly spaced, repeated monophasic calls. The female answered irregularly with long monophasic signals that immediately followed some of the calls. The males called with 3-8 repeated signals with long and variable call exchange intervals consisting of $9.1 \pm 1.1$ beats per signal (6-11) and intervals of $20.3 \pm 2.5 \mathrm{~ms}$. Call durations were $165.4 \pm 33.0 \mathrm{~ms}(90.8-$ 221.2), and the 3-8 irregularly repeated call signal durations were $8140.5 \pm 2951.3 \mathrm{~ms}$ (3351.814849.0). The number of beats per female answer signal was $30.7 \pm 3.2$ with intervals of $22.3 \pm$ $1.2 \mathrm{~ms}$. Answer durations were $662.9 \pm 68.48$ ms (585.7-716.4). The $\hat{o}_{\mathrm{C}^{-}}+9$ exchange interval was $159.9 \pm 38.4 \mathrm{~ms}$. The interbeat interval call pattern was approximately even and decreased from $20.2 \mathrm{~ms}$ (i1) to a minimum of $20.1 \mathrm{~ms}$ (i2), changed little over the next 6 intervals (i3-i8), then increased slightly to $21.7 \mathrm{~ms}$ (i9), and finally decreased to $20.6 \mathrm{~ms}$ (i10) (Fig. 22). The repeated call $\left(\hat{\sigma}_{\mathrm{C}}-\hat{\sigma}_{\mathrm{C}}\right)$ exchange interval pattern increased irregularly (Fig. 23), first decreasing from $1629.2 \mathrm{~ms}$ (il) to a minimum of $1583.8 \mathrm{~ms}$ (i2) and then increasing irregularly to a maximum of $1929.9 \mathrm{~ms}$ (i7). The answer interval pattern was approximately even (not charted), first decreasing from $23.8 \mathrm{~ms}$ (i1) to $21.9 \mathrm{~ms}$ (i2), continuing approximately evenly to interval $30(23.8 \mathrm{~ms})$, and finally increasing to $26.6 \mathrm{~ms}$ (i31) and $28.3 \mathrm{~ms}(\mathrm{i} 32)(n=1)$.

Oregon Gulch: One hundred thirty-seven calls were recorded from five $1-5 \mathrm{~d}$ males at 21.1-21. $6^{\circ} \mathrm{C}$. Males from this location also signaled with repeated monophasic calls. Although a female was present in the recording chamber next to each male, no answers were recorded. The males called with 2-9 repeated signals, $9.1 \pm 1.1$ beats per signal (7-12), and $22.8 \pm 1.3 \mathrm{~ms}$ intervals. Call durations were $212.3 \pm 24.8 \mathrm{~ms}(136.3-257.8)$, and the 2-9 repeated call durations were $2023.8 \pm 531.4$ ms (2059.1-4179.7).

The Oregon Gulch call interval pattern was approximately even (Fig. 24). It first decreased from $22.7 \mathrm{~ms}$ (i1) to a minimum of $22.6 \mathrm{~ms}$ (i2), increased irregularly to a maximum of $23.5 \mathrm{~ms}$ (i10), and finally decreased to $23.4 \mathrm{~ms}$ (i11). The repeated call $\left(\hat{\sigma}_{\mathrm{C}}-\hat{\sigma}_{\mathrm{C}}\right)$ exchange interval pattern increased irregularly (Fig. 25) from a minimum of $1731.3 \mathrm{~ms}$ (il) to a maximum of $2529.0 \mathrm{~ms}$ (i8). These results compared well with the Campbell Creek individuals, except that the Oregon Gulch individuals had slightly longer intergroup intervals (Table 2). The slight differences between individuals from the 2 locations may be attributed to slight differences in either age or temperature.

Stewart et al. (1991) first described the call for this species from 2 Napa County males as monophasic (Table 1), with no indication of repeated call signals. In that study, the number of beats per individual male call resembled the upper range of the observations made here (Table 3). Interbeat intervals reported in Stewart et al. (1991) were approximately half the duration of those reported here $(12.9 \pm 0.8$ ms vs. $20.3 \pm 2.5 \mathrm{~ms}$, respectively). 


\section{Discussion}

This study has employed a new technique to describe the variable signal types produced by stonefly drumming behavior. In all but one of the previous studies, the beat- and group intervals were summarized into a single entry listed in a table. Tierno De Figueroa et al. (2000) first presented the varied beat-interval call pattern of Isoperla curtata Navás using a box-andwhisker chart that illustrated the within-interval variation and entire-signal variation over 1-27 intervals. Using interval patterns will allow researchers to critically and statistically compare drumming characters between individuals and among populations, respectively. Greater uniformity in future experiments should decrease the number of observations (recordings) per individual and increase the number of individuals to describe population drumming characters. Furthermore, only reared (or at least field-collected and teneral) adult individuals with known ages at the time of recording, should be compared with other adults of the same age. Comparison of the drumming characters of adults 1-3 days old should be avoided except as needed for a first description of a species' drumming signals and should not be part of an experimental test. Finally, researchers must limit the amount of environmental variation at recording, such as temperature, which is suspected to influence the drumming characters of poikilothermic stoneflies. Perhaps placing the drumming recording chamber in another climate-controlled chamberwhere light intensity, humidity, and temperature can be regulated-would limit the effect of these potentially important variables.

Some of the species in this study have had updates to their previously reported drumming characters. This was expected, since newer, technologically advanced methods were employed. Ace of Wav software (Polyhedric Software, http://www.polyhedric.com/), which facilitates accurate and precise interval measurement in millisecond units, has replaced the storage oscilloscope and handmade ruler method of past studies. The increase in accuracy and precision of interval measurements, and recent increases to the number of species known to drum, have challenged the original definitions of the known drumming signals, including monophasic, varied beat-interval, diphasic, and grouped types (Stewart 2001, Stew- art and Sandberg 2006). In past studies, monophasic signal intervals were not thought to vary, or, if they did, the variation was poorly described. This study has attempted to improve the definition of monophasic signals by adding a limit of $10 \mathrm{~ms}$ to the allowable variation. This refinement will help future researchers identify the cutoff for monophasic patterns and the beginning of varied beat-interval patterns. A future improvement to the definition of the diphasic drumming pattern is also warranted due to its highly variable intervals and close resemblance to highly variable varied beat-interval patterns. This refinement will require future experiments at the individual and population levels with careful measurements of stonefly age and ambient conditions for each drumming event.

\section{ACKNOWLEDGMENTS}

Acknowledgment for over 4 years of help field-collecting live stoneflies and for direct assistance in writing this report go to Jim Harrington, Stephanie Lopez, Shawn McBride, Pete Ode, Dan Pickard, Andy Rehn, Austin Brady Richards, Joe Slusark, Jennifer York, and the remaining Aquatic Bioassessment Lab (CADFG) personnel. Special thanks to Allison Brigham, who first suggested interval patterns would be best displayed graphically using mean, standard deviation, and range data sets. Special thanks also to Jon Lee for assistance in field collection and species identifications. Finally, much appreciation to 2 anonymous reviewers, Toni Pilcher (WNAN copyediting intern), and Jill Bickham (WNAN production intern) for vastly improving this aritcle.

\section{Literature Cited}

AleXander, K.D., AND K.W. Stewart. 1997. Further considerations of mate searching behavior and communication in adult stoneflies (Plecoptera); First report of tremulation in Suwallia (Chloroperlidae). Pages 107112 in P. Landolt and M. Satori, editors, Ephemeroptera and Plecoptera biology-ecology-systematics. Mauron + Tinguely and Lachat SA, Fribourg.

Maketon, M., and K.W. SteWART. 1984. Further studies of the drumming behavior of North American Perlidae (Plecoptera). Annals of the Entomological Society of America 77:770-777.

1988. Patterns and evolution of drumming behavior in the stonefly families Perlidae and Peltoperlidae. Aquatic Insects 10:77-98.

Maketon, M., K.W. Stewart, B.C. Kondratieff, and R.F. KiRCHNER. 1988. New descriptions of drumming and evolution of the behavior in North American 
Perlodidae (Plecoptera). Journal of the Kansas Entomological Society 61:161-168.

SANDBERG, J.B. 2009. Vibrational communication (drumming) of the western Nearctic stonefly genus Hesperoperla (Plecoptera: Perlidae). Illiesia 5(13): $146-155$.

Sandberg, J.B., and K.W. Stewart. 2003. Continued studies of drumming in North American Plecoptera; evolutionary implications. Pages 73-81 in E. Gaino, editor, Research update on Ephemeroptera and Plecoptera. University of Perugia, Perugia, Italy.

2005. Vibrational communication (drumming) of the Nearctic stonefly genus Isogenoides (Plecoptera: Perlodidae). Transactions of the American Entomological Society 131(1+2):111-130.

. 2006. Continued studies of vibrational communication (drumming) of North American Plecoptera. Illiesia 2(1):1-14.

SteWART, K.W. 1994. Theoretical considerations of mate finding and other adult behaviors of Plecoptera. Aquatic Insects 16:95-104.

2001. Vibrational communication (drumming) and mate-searching behavior of stoneflies (Plecoptera); evolutionary considerations. Pages 217-225 in E. Dominguez, editor, Trends in research in Ephemeroptera and Plecoptera. Kluwer Academic/Plenum Publishers.

Stewart, K.W., R.L. Bottorff, A.W. Knight, and J.B. Moring. 1991. Drumming of four North American Euholognathan stonefly species, and a new complex signal pattern in Nemoura spiniloba Jewett (Plecoptera: Nemouridae). Annals of the Entomological Society of America 84:201-206.

Stewart, K.W., and J.B. Sandberg. 2004. Description of the nymph and drumming calls of Megaleuctra complicata Claassen (Plecoptera: Leuctridae); evolution of drumming in Leuctridae. Aquatic Insects 26: 123-129.

2006. Vibrational communication and mate searching behavior in stoneflies. Pages 179-186 in S. Drosopoulos and M. Claridge, editors, Insect sounds and communication: physiology, ecology and evolution. CRC Press, Boca Raton/London/New York.

Stewart, K.W., S.W. SzczYTKo, B.P. StakK, and D.D. ZEIGLER. 1982. Drumming behavior of six North American Perlidae (Plecoptera) species. Annals of the Entomological Society of America 75:549-554.

Stewart, K.W., and D.D. Zeigler. 1984. Drumming behavior of twelve North American stonefly (Plecoptera) species; first descriptions in Peltoperlidae, Taeniopterygidae, and Chloroperlidae. Aquatic Insects 6:49-61.

SzCZYTKO, S.W., and K.W. SteWart. 1979. Drumming of four western Nearctic Isoperla (Plecoptera) species. Annals of the Entomological Society of America 72: $781-786$.

Tierno De Figueroa, J.M., J.M. LuZÓn-Ortega, and A. SÁncheZ-ORTEGa. 2000. Male calling, mating and oviposition in Isoperla curtata (Plecoptera: Perlodidae). European Journal of Entomology 97:171-175.

Zeigler, D.D., AND K.W. SteWart. 1977. Drumming behavior of eleven Nearctic stonefly (Plecoptera) species. Annals of the Entomological Society of America 70:495-505.

1985. Drumming behavior of five stonefly (Plecoptera) species from central and western North America. Annals of the Entomological Society of America 78: $717-722$

Received 28 October 2010 Accepted 5 April 2011 